\title{
Erst das Vergnügen, dann die Arbeit
}

\section{Interprofessioneller Gesundheitskongress am 20. und 21. April in Dresden von Springer Medizin und Springer Pflege}

") „Haben Sie heute schon an sich gedacht?" - diese Frage wird Dr. Ilona Bürgel, Diplom-Psychologin, in Dresden den rund 800 Teilnehmenden des 6. Interprofessionellen Gesundheitskongresses stellen. Der Kongress richtet sich an Mediziner, Pflegefachkräfte und Angehörige anderer Gesundheitsberufe - Menschen also, die es gewohnt sind, zunächst an andere zu denken. Die beiden Springer-Bereiche Springer Medizin und Springer Pflege veranstalten den Kongress, der am 20. und 21. April 2018 im Internationalen Congress Center Dresden direkt am Ufer der Elbe stattfindet.

Erst die Arbeit, dann das Vergnügen. Dieser Spruch gelte für die neue Arbeitswelt nicht mehr, betont Bürgel. „Weil die Anforderungen immer weiter wachsen, müssen wir den Satz um- drehen: Erst wenn es mir gut geht, kann ich mehr leisten." Im Stress neigen wir dazu, uns auf das Negative zu fokussieren und einen Tunnelblick zu entwickeln. „In dieser Stressfalle sind wir wenig kreativ, sehen keine Lösungen und können keine guten Entscheidungen treffen“, sagt die Psychologin, die als Referentin für Positive Psychologie Unternehmen berät. Daher gilt: Das negative Denken bewusst stoppen und umschalten. Das Universitätsklinikum Dresden hat dazu das Werkzeug „Die schöne Woche“ entwickelt: Jede Führungskraft erzählt zu Beginn des wöchentlichen Meetings, was in der vergangenen Woche gut gelaufen ist. „Das schafft eine Atmosphäre, in der man gut arbeiten kann“, so Bürgel.

Gesund bleiben im Gesundheitsberuf gehört zu den interprofessionellen
Modulen des Kongresses genauso wie die Veranstaltungen zu Palliative Care, Diabetes, Risikomanagement oder Digitalisierung im Gesundheitswesen. Daneben gibt es spezielle Module für Mediziner, Medizinische Fachangestellte und Pflegefachkräfte. Für im Notfall- und Rettungsbereich Tätige bietet der Deutsche Berufsverband Rettungsdienst e.V. nach der erfolgreichen Premiere 2017 wieder das Notfall Spezial an, dieses Mal zu Kardiopulmonaler Reanimation. Der Sächsische Pflegerat und die Sächsische Landesärztekammer sind zwei der zahlreichen Kooperationspartner des Kongresses. Hauptsponsor ist wieder Bristol-Myers Squibb.

\section{http://gesundheitskongresse.de/ dresden/2018/}

\section{Kommunen sind gefordert}

\section{Gesundheits- und Pflegeangebote müssen dort zu finden sein, wo die Menschen leben, fordert NRW-Gesundheitsminister Laumann.}

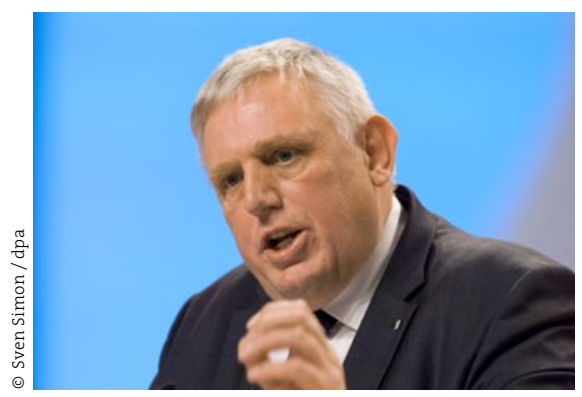

") Die Sicherstellung der gesundheitlichen und pflegerischen Versorgung von alten Menschen stellt nicht nur die Gesundheitspolitik vor große Herausforderungen. Auch die Kommunalpolitik muss sich mit dem Thema befassen, fordert der nordrhein-westfälische Gesundheitsminister Karl-Josef Laumann (CDU). Städte und Gemeinden müssten sich Gedanken machen, wie sie Angebote für diese Gruppe sicherstellen können, sagte Laumann auf der Veranstaltung „Im Alter medi- zinisch gut versorgt: Hürden abbauen. Übergänge gestalten“ der Ärztekammer Westfalen-Lippe.

„Wir haben die Aufgabe, die Leistungen des Gesundheits- und des Pflegesystems dahin zu bringen, wo die Menschen leben“, betonte er. Altersgerechte Wohnungen, Kurzzeitpflegeplätze und weitere Angebote müssten mit Blick auf die vorhandene Infrastruktur konzipiert werden. Die Kommunalpolitik müsse sich um solche Fragen kümmern. Städte und Gemeinden nutzen ihr Potenzial nicht aus, findet Laumann. Im Internetzeitalter sollte es den Verwaltungen möglich sein, im Netz eine Seite anzubieten, in der Pflegeheime täglich freie Plätze in der Kurzzeitpflege melden. Dann müssten Angehörige und niedergelassene Ärzte im Bedarfsfall nicht erst aufwändig suchen.

Der Blickwinkel auf einzelne Versorgungsbereiche hilft seiner Ansicht nach nicht weiter. „Menschen leben nicht in einem Sektor." In die Überlegungen einbezogen werden müssten die ambulanten und stationären Strukturen, das Ehrenamt und das familiäre Umfeld. Wichtig ist ihm, dass die alten Menschen selbst entscheiden, wo sie leben wollen. „Die Forderung ambulant vor stationär werden Sie von mir nie hören."

Angesichts des Mangels an Ärzten und Pflegefachkräften ist für ihn klar, dass die Versorgung in einer älter werdenden Gesellschaft nicht allein auf einer professionellen Ebene gemeistert werden kann. „Man muss die Professionalität mit dem Umfeld der Menschen zusammenbringen", so der Minister. Laumann plädierte dafür, angesichts der Vielfalt der gesundheitlichen und pflegerischen Angebote nicht zu sehr auf die staatliche Planung zu hoffen. Vieles werde besser, wenn man es dem Markt überlässt. „Warum haben wir zu wenige Pflegekräfte? Weil wir über die Pflegeschulen geplant haben." 